\title{
Analysis of Relativity Premium in Bonus-Malus System Based on Optimal Linear Method
}

\author{
Yu Chen and Long Li \\ Department of Statistics and Finance, School of Management, University of Science and Technology of China, Hefei 230026, China \\ Correspondence should be addressed to Long Li; cdll@mail.ustc.edu.cn
}

Received 3 June 2014; Revised 13 August 2014; Accepted 1 September 2014; Published 29 September 2014

Academic Editor: Daniela Boso

Copyright (c) 2014 Y. Chen and L. Li. This is an open access article distributed under the Creative Commons Attribution License, which permits unrestricted use, distribution, and reproduction in any medium, provided the original work is properly cited.

\begin{abstract}
A bonus-malus system plays a very important role in actuarial mathematics through determining its relativity premium, which is extensively used in automobile insurance. There are many ways including Bayesian estimator and ordinary linear estimator to calculate the relativity premium. There is no doubt that Bayesian estimator is the most accurate estimator; however, it is undesirable for commercial purposes for its rather irregular pattern. This paper aims to introduce an optimal linear estimator for relativity premium, which has a simple pattern and is obtained under the quadratic loss function such that the result is close to Bayesian method. The Loimaranta efficiency of such an optimal linear estimator has been studied and compared with the two methods mentioned above.
\end{abstract}

\section{Introduction}

Bonus-malus system (BMS) is an important research field in modern actuary. In insurance, BMS is a system that adjusts the premium paid by a policyholder according to his individual claim history. Bonus usually is a discount in the premium which is given on the renewal of the policy if no claim is made in the previous year. Malus is an increase in the premium if there is a claim in the previous year. Bonusmalus systems are very common in vehicle insurance. And it is a topic of interest of how to compute the relativity premium of a bonus-malus system.

Consider a bonus-malus system containing $s$ levels, labeled 1 to $s$, and the next level is determined by the current level and the number of claims reported during the current period. If the number of claims in different periods is independent, then the trajectory of a given policyholder will be a Markov chain. To deal with the heterogeneity of BMS, it is natural to consider that the claim numbers $N$ caused by the policyholder are assumed to be mixed Poisson distributed. More precisely, $N$ has conditional discrete probability function of the following form:

$$
P(N=k \mid \Theta=\theta)=\frac{e^{-\lambda \theta}(\lambda \theta)^{k}}{k !}, \quad k=0,1,2, \ldots,
$$

where $\lambda$ and $\Theta$ represent the mean frequency and random risk effect, respectively. The random effect $\Theta$ represents the risk proneness of the policyholder, that is, unknown risk characteristics having a significant impact on the occurrence of claims. $F_{\Theta}(\theta)$ is the distribution function of $\Theta$. According to Norberg [1], the probability mass function associated with level $K$ for a given policyholder is

$$
P[K=k]=\int_{0}^{\infty} \pi_{k}(\lambda \theta) d F_{\Theta}(\theta),
$$

and the Bayesian relativity premium $r_{k}^{\text {Bays }}$ under the quadratic loss function is

$$
r_{k}^{\text {Bays }}=\mathbb{E}(\Theta \mid K=k)=\frac{\int_{0}^{\infty} \theta \pi_{k}(\lambda \theta) d F_{\Theta}(\theta)}{\int_{0}^{\infty} \pi_{k}(\lambda \theta) d F_{\Theta}(\theta)},
$$

where $F_{\Theta}(\theta)$ is the distribution function of $\theta$ and $\pi_{k}(\vartheta)$ is the stationary probability for a policyholder with level $k$. The idea of Bayesian way is to attach a relativity premium to the randomly picked policyholder according to his relative risk parameter and the closeness or relativity is measured by Bayesian methods. Obviously the Bayesian estimator has a rather complex and irregular pattern and one cannot see that the relativity premium is regularly increasing according to the level $k$. 
Many authors have discussed the problem of how to design an optimal bonus-malus system. For example, Lemaire and $\mathrm{Zi} \mathrm{[2]} \mathrm{compared} \mathrm{the} \mathrm{validity} \mathrm{of} 30$ bonus-malus systems by four different tools, such as the relative stationary average premium level, the coefficient of variation of the insured's premiums, the efficiency of the bonus-malus system, and the average optimal retention. Lemaire [3] suggested that the claim frequency as negative binomial distributed and used the quadratic loss function to study the bonus-malus system. Walhin and Paris [4] discussed the problem mainly using a finite Poisson mixture distribution as the claim frequency distribution. All those authors took the claim frequency as the most important factor and used the Bayesian estimator. The Bayesian estimator not only presents a rather irregular pattern but also may result unfairly without taking the severity of each claim into account. Frangos and Vrontos [5] designed an optimal bonus-malus system by taking both the frequency and the severity of the claim into consideration, and Mahmoudvand and Hassani [6] developed the system to a generalized form with a frequency and a severity component based both on the a priori and on the a posteriori classification criteria. And this makes sense for the system allowing for both the frequency and severity to distinguish different premiums with equal claim times and it is fair from the point of insured.

Obviously, the Bayesian estimator has a rather complex and irregular pattern. In consideration of the drawbacks of the Bayesian relativity premium, Gilde and Sundt [7] suggested to smooth $r_{k}^{\text {Bays }}$ by a linear pattern; that is, $r_{k}^{\operatorname{Lin}}=$ $\alpha_{\text {lin }}+\beta_{\text {lin }} K, K=1,2, \ldots, s$, and obtained the parameter values of $\alpha_{\text {lin }}$ and $\beta_{\text {lin }}$ under the condition of minimizing $\mathbb{E}\left[\left(\Theta-r_{k}^{\mathrm{Lin}}\right)^{2}\right]$. The solution of $r_{k}^{\mathrm{Lin}}$ can be easily gotten as follows:

$$
r_{k}^{\mathrm{Lin}}=1+\frac{\operatorname{cov}(K, \Theta)}{\operatorname{var}(K)}(k-E[K])
$$

where $\operatorname{cov}(K, \Theta)=\left(\sum_{k=1}^{s} k \int_{0}^{\infty} \theta \pi_{k}(\lambda \theta) d F_{\Theta}(\theta)-E[K]\right)$ is the covariance of level $k$ and risk parameter $\Theta$ and $E[K]$ and $\operatorname{var}(K)$ are the expectation and variance of level $k$. With this estimator derived from the quadratic loss function of $\Theta$ and $r_{k}^{\mathrm{Lin}}$, we can compute the relativity premium easily and the linear pattern has the increasing property associate with the level $k$. But it still does not guarantee fairness from the insurers' side because the linear form imposes the same degree when the bonus-malus system penalizes or rewards a certain policyholder.

Though assuming that the frequency or severity is differently distributed, successively, researchers used Bayesian estimator or linear estimator to calculate the relative premium when designing or developing bonus-malus system. No matter how well the heterogeneity of auto insurance is depicted from the insured's side through the frequency or severity distribution, however, the behavior from the insurers' side is not represented that well. Thus the relativity premiums calculated by Bayesian estimator as equation (3) shows or by the Ordinary Linear estimator as equation (4) shows, miss the insurers' point. On the other hand, the Bayesian estimator has a rather complex pattern for commercial use and an irregular pattern for merit rating mechanism; that is, the relationship between the relativity premium $r_{k}^{\text {Bays }}$ and level $k$ is implicit, though it is most accurate. And the Ordinary Linear estimator smoothed from the Bayesian estimator cannot be as accurate as the Bayesian method and is not that fair due to the same degree or effect imposed on a policyholder when insurers do penalizing and rewarding, though it is easy to compute. Given for this, this paper introduces a new estimator, namely, the Optimal Linear estimator which we expect to be close to Bayesian estimator to calculate the relativity premium. The Optimal Linear estimator, derived from the surplus capital equation from the point of insurers and with the linear pattern by smoothing the Bayesian estimator under the quadratic loss function of surplus capital, will not only be easily used for computing but also be much closer to the Bayesian estimator than the Ordinary Linear estimator. And the Optimal Linear estimator is regularly increasing according to the level $k$ too. Then, we will show how the new method performs better than the Ordinary Linear estimator by introducing the Loimaranta efficiency as a tool for comparing. The comparison includes the demonstration of the Hong Kong auto insurance market and the simulation of a bonus-malus system allowing for claim amounts.

The organization of this paper is as follows. Section 2 introduces a simple method, namely, the Optimal Linear estimator, which can be used to compute the relativity premium in a steady state bonus-malus system. A comparison for the three estimators' efficiency based on Loimaranta efficiency is discussed in Section 3. And some simulations and demonstrations allowing for severity of the claims of each policyholder are also presented in Section 3. Then, the conclusion remarks come.

\section{The Optimal Linear Relativity}

As mentioned above, the BMS discussed in this paper has $s$ levels, labeled 1 to $s$, with $r_{k}$ as the relativity premium associated with level $k$. The problem addressed here is to determine the solution of Optimal Linear relativity premium $r_{k}^{\mathrm{Opt}}$. In order to go further, suppose that $M_{k}(t), k=1,2, \ldots, s$, represents the number of policyholders in level $k$ at time $t$ and $u_{0}$ is the initial capital and $B$ is the benchmark of the premium. Then, the surplus capital of the insurer at time $t$ can easily be obtained as

$$
\varphi(t)=u_{0}+U(t)-S(t)
$$

where $U(t)=B \sum_{k=1}^{s} r_{k} M_{k}(t)$ denotes the whole premium incomes and $S(t)=\sum_{i=1}^{N(t)} X_{i}$ is the amount of all the claims at time $t$. Similarly, we can naturally get the whole premium incomes

$$
U^{\text {Bays }}(t)=B \sum_{k=1}^{s} r_{k}^{\text {Bays }} M_{k}(t)
$$

associated to the Bayesian relativity and

$$
U^{\mathrm{Opt}}(t)=B \sum_{k=1}^{s} r_{k}^{\mathrm{Opt}} M_{k}(t)
$$


associated to the Optimal Linear relativity. The question of questions is to smooth the Bayesian relativity $r_{k}^{\text {Bays }}$ and achieve the Optimal Linear relativity which has an explicit linear pattern, denoted as

$$
r_{k}^{\mathrm{Opt}}=\alpha_{\mathrm{opt}}+\beta_{\mathrm{opt}} K
$$

We embark on this issue from the point of the surplus capital of the insurer and achieve the parameter values of $\alpha_{\mathrm{opt}}$ and $\beta_{\mathrm{opt}}$ by minimizing the quadratic loss function $E\left[\left(\varphi(t)^{\text {Bays }}(t)-\varphi(t)^{\mathrm{Opt}}(t)\right)^{2}\right]$.

To do this, we give priority to the whole premium incomes $U(t)$ and assume the initial capital $u_{0}$ and the amount of all the claims $S(t)=\sum_{i=1}^{N(t)} X_{i}$ at time $t$ to be fixed since our purpose is to get the answer of $r_{k}^{\mathrm{Opt}}$, and this assumption is suitable here.

Proposition 1. Consider a bonus-malus system having s levels, labeled 1 to $s$, with $r_{k}^{\text {Bays }}$ and $r_{k}^{\text {Opt }}=\alpha_{o p t}+\beta_{o p t} K, K=1$, $2, \ldots s$, being the Bayesian relativity and the Optimal Linear relativity, respectively; then, the constrained minimization $\min E\left[\left(\varphi(t)^{\text {Bays }}(t)-\varphi(t)^{\text {Opt }}(t)\right)^{2}\right]$ holds if and only if

$$
\begin{aligned}
\alpha_{o p t}= & \left(E\left(U^{\text {Bays }}(t) M(t)\right) E\left(M^{\star}(t)^{2}\right)\right. \\
& \left.-E\left(U^{\text {Bays }}(t) M^{\star}(t)\right) E\left(M(t) M^{\star}(t)\right)\right) \\
\times & \left(E\left(M(t)^{2}\right) E\left(M^{\star}(t)^{2}\right)-\left[E\left(M(t) M^{\star}(t)\right)\right]^{2}\right)^{-1} \\
\beta_{o p t}= & E\left(U^{\text {Bays }}(t) M^{\star}(t)\right) E\left(M(t)^{2}\right) \\
& \left.-E\left(U^{\text {Bays }}(t) M(t)\right) E\left(M(t) M^{\star}(t)\right)\right) \\
\times & \left(E\left(M(t)^{2}\right) E\left(M^{\star}(t)^{2}\right)-\left[E\left(M(t) M^{\star}(t)\right)\right]^{2}\right)^{-1},
\end{aligned}
$$

where $M(t)=\sum_{k=1}^{s} M_{k}(t)$ and $M^{\star}(t)=\sum_{k=1}^{s} k M_{k}(t)$.

Proof. Since the benchmark of the premium $B$ is a constant and $S(t)=\sum_{i=1}^{N(t)} X_{i}$ is assumed to be fixed, obviously, we will have

$$
\begin{aligned}
\phi & =E\left[\left(\varphi(t)^{\text {Bays }}(t)-\varphi(t)^{\text {Opt }}(t)\right)^{2}\right] \\
& =E\left[\left(U^{\text {Bays }}(t)-U^{\text {Opt }}(t)\right)^{2}\right] \\
& =E\left[\left(\sum_{k=1}^{s} r_{k}^{\text {Bays }} M_{k}(t)-\sum_{k=1}^{s} r_{k}^{\text {Opt }} M_{k}(t)\right)^{2}\right] \\
& =E\left[\left(\sum_{k=1}^{s}\left(r_{k}^{\text {Bays }}-\alpha-\beta K\right) M_{k}(t)\right)\right]^{2} .
\end{aligned}
$$

Differentiating this equation with respect to $\alpha$ and $\beta$, we obtain

$$
\begin{aligned}
& \frac{\partial \phi}{\partial \alpha} \\
& \quad=2 E\left\{\left[\sum_{k=1}^{s}\left(r_{k}^{\text {Bays }}-\alpha-\beta K\right) M_{t}(t)\right]\left[-\sum_{k=1}^{s} M_{t}(t)\right]\right\}=0
\end{aligned}
$$

$\frac{\partial \phi}{\partial \beta}$

$$
=2 E\left\{\left[\sum_{k=1}^{s}\left(r_{k}^{\text {Bays }}-\alpha-\beta K\right) M_{t}(t)\right]\left[-\sum_{k=1}^{s} K M_{t}(t)\right]\right\}=0 .
$$

Simplifying the two equations above, then, we have

$$
\begin{gathered}
E\left(U^{\text {Bays }}(t) M(t)\right)-\alpha E\left(M(t)^{2}\right)-\beta E\left(M(t) M^{\star}(t)\right)=0 \\
E\left(U^{\text {Bays }}(t) M^{\star}(t)\right)-\alpha E\left(M(t) M^{\star}(t)\right)-\beta E\left(M^{\star}(t)^{2}\right)=0 .
\end{gathered}
$$

Combine and solve the two equations, then we obtain the proposition we need to prove.

From the solution procedure of parameter values of $\alpha_{\text {opt }}$ and $\beta_{\text {opt }}$, we see that the Optimal Linear relativity discussed here is not only just a linear pattern by minimizing risk function of relativity and risk parameter but also a regularly increasing linear pattern after giving enough thought to the number of policyholders associated to each level. By taking into consideration both the surplus capital equation of the insurer and the distribution of the number of policyholders in each level, we believe that the Optimal Linear relativity addressed in this paper will be closer to the Bayesian estimator and correspond to a better bonus-malus system compared with the Ordinary Linear relativity. And also, with an explicit pattern, the Optimal Linear relativity can be computed easily.

\section{Comparison of the Three Approaches}

3.1. Loimaranta Efficiency as a Tool to Compare. A good bonus-malus system should have this merit that the premium paid by the policyholders subject to bonus-malus scales is increasing as the expected claim frequency is increasing. The greater the increasing rate is, the better the bonus-malus system is. And the rate of increase is related to the concept of elasticity which measures one factor's response to a change of the other. Loimaranta efficiency [8] is just a good case to measure how the bonus-malus system responds to a change in the risk of a driver. The average relativity, denoted as $\bar{R}(\vartheta)$, has the following formula once each policyholder ultimately stabilizes around an equilibrium level:

$$
\bar{R}(\vartheta)=\sum_{k=1}^{s} r_{k} \pi_{k}(\vartheta)
$$


And the Loimaranta efficiency, denoted as $\Xi(\vartheta)$, is then defined as the elasticity of the average relativity and has the following formula:

$$
\Xi(\vartheta)=\frac{d \ln (\bar{R}(\vartheta))}{d \ln (\vartheta)} .
$$

Loimaranta efficiency measures how the average relativity responds to the change of the mean claim frequency and ideally the efficiency should be close to 1 for the most common values of $\lambda$. The larger the efficiency is, the better the system is. We have given the definition of Loimaranta efficiency for a certain claim frequency so far. However, it is not enough for deep discussion, so we calculate the Loimaranta efficiency which is a function of the annual expected claim frequency where $\lambda \in(0,0.8)$ for explicit comparison. Now that we introduce this useful tool for comparison, we will compare three estimating relativities, those are the Bayesian relativity and the Ordinary Linear relativity and the Optimal Linear relativity, by using some simulations and demonstrations.

3.2. Comparison with Demonstration. Let us take the autoinsurance market of Hong Kong as an example. The bonusmalus system in Hong Kong contains 6 levels, labeled 1 to 6 , and the starting level is the highest level 6 . The discount of per claim-free year is one level, and the penalty per claim reported is two levels. All the discounts will be lost and move to the highest level 6 after more than one claim is reported. The corresponding transition rules are displayed in Table 1.

According to the transition rules in Table 1, we obtain the corresponding transition matrix $P(\vartheta)$ under the occasion that the claim numbers $N$ are mixed Poisson distributed:

$$
P(\vartheta)=\left(\begin{array}{ccc}
p_{11}(\vartheta) & \cdots & p_{16}(\vartheta) \\
\vdots & \ddots & \vdots \\
p_{61}(\vartheta) & \cdots & p_{66}(\vartheta)
\end{array}\right),
$$

where $p_{k 1 k 2}(\vartheta)$, element of the matrix, denotes the transition probability from level $k_{1}$ to level $k_{2}$. The solution of this stochastic matrix $P(\vartheta)$ is

$$
\Pi^{T}(\vartheta)=e^{T}(I-P(\vartheta)+E)^{-1},
$$

where $e$ is a column vector of 1's and $E$ is the $s * s$ matrix with all entries 1.

For convenience, we follow Denuit et al.s [9] conclusion that the mean claim frequency $\hat{\lambda}$ equals 0.1426 which is gotten by using the maximum likelihood and assume that the random risk effect $\Theta$ is Gamma distributed, that is,
$\Theta \sim \Gamma(1,1)$. Suppose that the numbers of policyholders in each level $M(t)=\left(M_{1}(t), M_{2}(t), \ldots, M_{6}(t)\right)$ are independent and Poisson distributed with mean $\mu=0.95$. With those assumptions, we obtain corresponding parameter values displayed in Tables 2 and 3.

Now, we are ready for comparing those three estimating relativities by using Loimaranta efficiency after obtaining those data displayed in Tables 2 and 3. And we have Figure 1 associated to different mean claim frequency $\lambda$ which belongs to the interval $(0,0.8)$.

Seeing from Figure 1, the Loimaranta efficiency of the Bayesian relativity is the biggest and the efficiency of the Optimal Linear relativity is better than the Ordinary Linear relativity for the whole interval of $\lambda$. The conclusion presented in Figure 1 confirms to our expectation that the Bayesian relativity can be considered the most accurate and thus is the most efficient. As Figure 1 shows, the Optimal Linear estimator nearly gets the efficiency of Bayesian method. Besides, the Optimal Linear estimator has much better efficiency than the Ordinary Linear estimator. Theoretically speaking, the Bayesian method gets the results by using the a priori information of mean claim frequency, thus being the most accurate which for sure. And it is easy to understand that the Optimal Linear estimator obtained by minimizing $E\left[\left(\sum_{k=1}^{s}\left(r_{k}^{\text {Bays }}-r_{k}^{\text {Opt }}\right) M_{k}(t)\right)\right]^{2}$ has better efficiency than the Ordinary Linear estimator obtained by minimizing $\mathbb{E}\left[\left(\Theta-r_{k}^{\mathrm{Lin}}\right)^{2}\right]$ for $r_{k}^{\mathrm{Opt}}$ being much closer to Bayesian estimator than $r_{k}^{\text {Lin }}$.

\subsection{Comparison with Simulations Allowing for Claim Severity.} Consider a new bonus-malus system which has 6 levels, labeled 1 to 6 , and allows for both claim numbers and claim severity. Let random variable $X$ denote the claim amounts and satisfy the requirements of $p_{x}(a)=p(x \leq a), p_{x}(b)=$ $p(x \geq b)$, and $p_{x}(a, b)=p(a<x<b)$, where $a$ and $b$ are certain given claim amounts and meet $b>a>0$. Let random variable $n$ denote the numbers of claim and $p_{n}$ is the distribution function of $n$. The discount of per claimfree year is one level. Under the condition that one claim is reported, the penalty will be two levels if the claim amounts $x$ are smaller than $a$, and the penalty will be three levels if the claim amounts $x$ are between $a$ and $b$. All the discounts will be lost and the policyholder will transfer to the highest level 6 after two or more claims are reported or the claim amounts $x$ exceeds $b$. And the corresponding transition rules are displayed in Table 4.

Corresponding to the transition rules in Table 4, the transition matrix $P$ is

$$
P=\left(\begin{array}{cccccc}
p_{0} & 0 & p_{x}(a) * p_{1} & p_{x}(a, b) * p_{1} & 0 & p_{x}(b) * p_{1}+(1-p) \\
p_{0} & 0 & 0 & p_{x}(a) * p_{1} & p_{x}(a, b) * p_{1} & p_{x}(b) * p_{1}+(1-p) \\
0 & p_{0} & 0 & 0 & p_{x}(a) * p_{1} & \left(1-p_{x}(a) * p_{1}+(1-p)\right. \\
0 & 0 & p_{0} & 0 & 0 & 1-p_{0} \\
0 & 0 & 0 & p_{0} & 0 & 1-p_{0} \\
0 & 0 & 0 & 0 & p_{0} & 1-p_{0}
\end{array}\right)
$$


TABLE 1: Transition rules of BMS in Hong Kong.

\begin{tabular}{lccc}
\hline Staring level & \multicolumn{3}{c}{ Level occupied if } \\
& $n=0$ & $n=1$ & $n \geqq 2$ \\
\hline 1 & 1 & 3 & 6 \\
2 & 1 & 4 & 6 \\
3 & 2 & 5 & 6 \\
4 & 3 & 6 & 6 \\
5 & 4 & 6 & 6 \\
6 & 5 & 6 & 6 \\
\hline
\end{tabular}

TABle 2: Parameters of the linear estimator (Hong Kong).

\begin{tabular}{lcccc}
\hline & $\alpha_{\text {lin }}$ & $\beta_{\text {lin }}$ & $\alpha_{\text {opt }}$ & $\beta_{\text {opt }}$ \\
\hline Hong Kong & 0.2577 & 0.4123 & 0.3284 & 0.3985 \\
\hline
\end{tabular}

TABLE 3: Relativities and probability associated to each level (Hong Kong).

\begin{tabular}{lcccc}
\hline Level & $P[K=k]$ & $r_{k}^{\text {Bays }}$ & $r_{k}^{\text {Lin }}$ & $r_{k}^{\text {Opt }}$ \\
\hline 1 & 0.6994 & 0.6552 & 0.6700 & 0.7269 \\
2 & 0.0736 & 1.2848 & 1.0823 & 1.1254 \\
3 & 0.0895 & 1.3999 & 1.4946 & 1.5239 \\
4 & 0.0439 & 1.9996 & 1.9069 & 1.9224 \\
5 & 0.0458 & 2.2873 & 2.3192 & 2.3209 \\
6 & 0.0467 & 2.7146 & 2.7315 & 2.7194 \\
\hline
\end{tabular}

For convenience but without loss of generality, we set $p_{x}(a)=$ $0.05, p_{x}(a, b)=0.9$, and $p_{x}(b)=0.05$ and $p_{x}(a)=$ $0.05, p_{x}(a, b)=0.05$, and $p_{x}(b)=0.9$. In both cases, we undergo our simulation with all the assumptions mentioned above; $\hat{\lambda}=0.1462, \Theta \sim \Gamma(1,1)$, the elements of $M(t)=$ $\left(M_{1}(t), M_{2}(t), \ldots, M_{6}(t)\right)$ are independent and are Poisson distributed with mean $\mu=0.95$.

Then in the first case, according to the proposition we have corresponding estimator values in Table 5 .

And the Loimaranta efficiency associated with those estimator values in Table 5 is shown in Figure 2.

The efficiency shown in Figure 2 is beyond our expectation to some extent. The Loimaranta efficiency of the Optimal Linear relativity can easily get close to Bayesian estimator and obviously performs much better than the Ordinary Linear relativity in the whole given interval of $\lambda$.

For the second case, we obtain the corresponding estimator values in Table 6 according to the proposition.

And the Loimaranta efficiency associated with those estimator values in Table 6 is shown in Figure 3.

Seeing Figure 3 and as expected, the efficiency of the Bayesian relativity is the biggest and also the efficiency of the Optimal Linear relativity is better than that of the Ordinary Linear relativity.

At this point, we have the conclusion that the Optimal Linear relativity is better than the Ordinary Linear relativity by the demonstration of the bonus-malus system in Hong Kong and the simulation for a new bonus-malus system allowing for claim severity, under the framework of Loimaranta efficiency. Yet the only downside is that the Optimal Linear relativity is not as close to the real value as

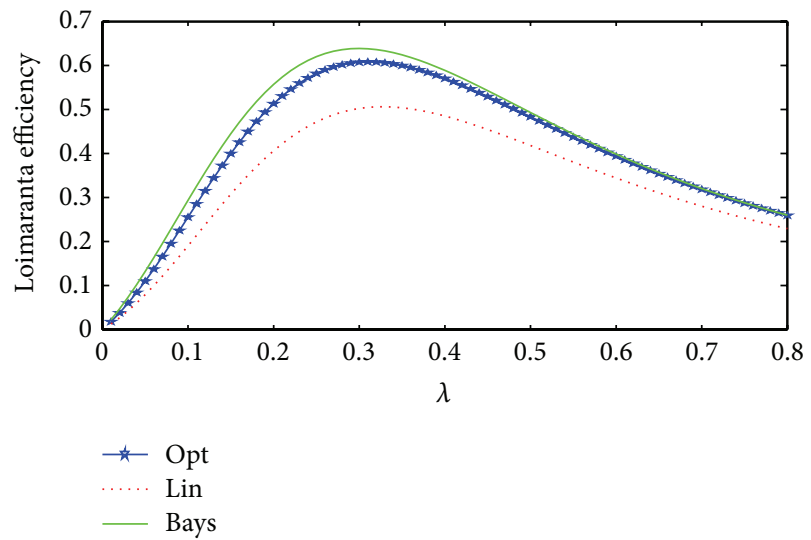

FIGURE 1

TABLE 4: Transition rules (simulation).

\begin{tabular}{lcccc}
\hline \multirow{2}{*}{ Starting level } & \multicolumn{4}{c}{ Levels occupied if } \\
& $n=0$ & $n=1, x \leq a$ & $n=1, a<x<b$ & Others \\
\hline 1 & 1 & 3 & 4 & 6 \\
2 & 1 & 4 & 5 & 6 \\
3 & 2 & 5 & 6 & 6 \\
4 & 3 & 6 & 6 & 6 \\
5 & 4 & 6 & 6 & 6 \\
6 & 5 & 6 & 6 & 6 \\
\hline
\end{tabular}

the Bayesian relativity is; however, it can perform much as well as the Bayesian estimator. Theoretically and statistically, the Bayesian method using the a priori information of mean claim frequency of policyholder works better which for sure. $r_{k}^{\mathrm{Opt}}$ obtained from the surplus capital equation and minimizing $E\left[\left(\sum_{k=1}^{s}\left(r_{k}^{\text {Bays }}-r_{k}^{\text {Opt }}\right) M_{k}(t)\right)\right]^{2}$ is a linear pattern by smoothing $r_{k}^{\text {Bays }}$ directly. $r_{k}^{\text {Lin }}$ obtained by minimizing $\mathbb{E}\left[\left(\Theta-r_{k}^{\mathrm{Lin}}\right)^{2}\right]$ is a linear pattern just by smoothing the random risk parameter $\Theta$. Based on the point that Bayesian method is the most accurate, the fact that the Optimal Linear estimator is much closer to Bayesian estimator and works better than the Ordinary Linear estimator is understandable. And, of course, this paper is just trying to enrich some kind of methods for relativity estimating and is limited to the standard of Loimaranta efficiency. How the Optimal Linear relativity performs needs further research. Taking all this condition, however, the Optimal Linear relativity would be a good method due to its relative accuracy and simplicity.

\section{Concluding Remarks}

How to determine the premium relativity comes to a long way and gets remarkable progress including building models and seeking ways to estimate those models. Because of the undesirable implicity and complexity of the Bayesian estimator and some drawbacks of the Ordinary Linear estimator, this paper develops a new estimating method by smoothing the Bayesian estimator in order to be much closer to Bayesian method. And this new estimating method, which started 


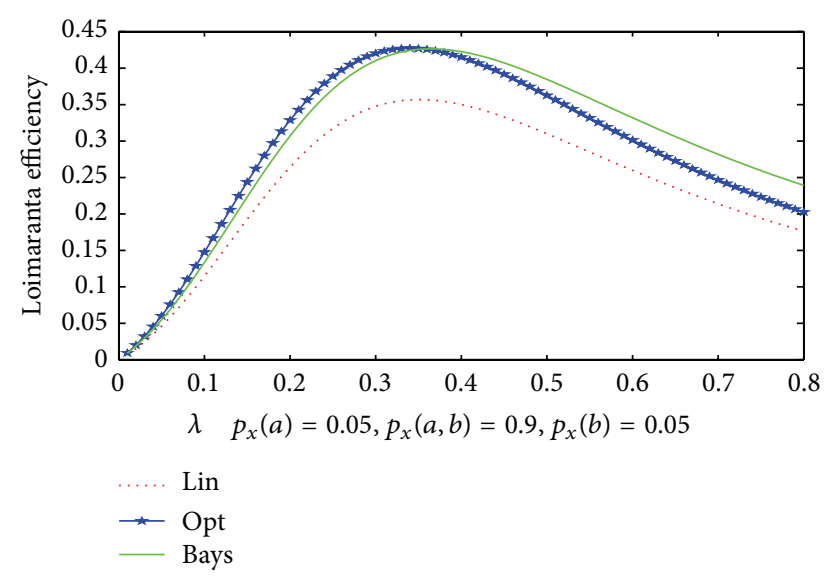

FIGURE 2

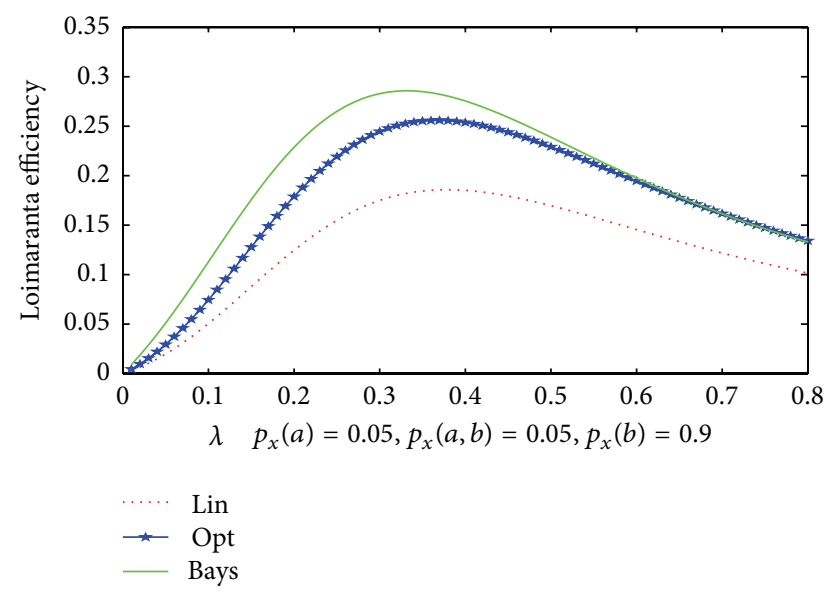

FIgURE 3

from the insurer's surplus capital function, is of great empirical significance for it can avoid the complexity of the Bayesian method and is more effective than the Ordinary Linear method. In practice, the proposed Optimal Linear method is used to analyze the Hong Kong automobile market and a constructed bonus-malus system allowing for severity and is judged under the frame of Loimaranta efficiency. Loimaranta efficiency, as a function of the expected claim frequency $\lambda$, shows that the proposed optimal method is superior to the Ordinary Linear method, especially apparently when $\lambda$ is in the interval $(0.2,0.4)$. For further research, the proposed method can be extended and applied to different kinds of autoinsurance market and can be checked by other tools, and the number of policyholders in each level, represented by the parameter $M_{k}(t)$, can be thought carefully and extended to other distributions.

\section{Conflict of Interests}

The authors declare that there is no conflict of interests regarding the publication of this paper.
TABLE 5: Estimator values when $p_{x}(a)=0.05, p_{x}(a, b)=0.9$ and $p_{x}(b)=0.05$.

\begin{tabular}{lcccccc}
\hline Level & 1 & 2 & 3 & 4 & 5 & 6 \\
\hline$P[K=k]$ & 0.1686 & 0.0855 & 0.1699 & 0.2506 & 0.1882 & 0.2028 \\
$r_{k}^{\text {Bays }}$ & 0.5719 & 0.7995 & 0.8180 & 0.8745 & 1.2253 & 1.4192 \\
$r_{k}^{\text {Lin }}$ & 0.6052 & 0.7386 & 0.8684 & 1.0000 & 1.1316 & 1.2632 \\
$r_{k}^{\text {Opt }}$ & 0.5534 & 0.7126 & 0.8718 & 1.0310 & 1.1902 & 1.3494 \\
\hline
\end{tabular}

TABLE 6: Estimator values when $p_{x}(a)=0.05, p_{x}(a, b)=0.05$, and $p_{x}(b)=0.9$.

\begin{tabular}{lcccccc}
\hline Level & 1 & 2 & 3 & 4 & 5 & 6 \\
\hline$P[K=k]$ & 0.1152 & 0.0601 & 0.1175 & 0.1775 & 0.2451 & 0.3275 \\
$r_{k}^{\text {Bays }}$ & 0.6127 & 0.8656 & 0.8893 & 0.9547 & 1.0415 & 1.1496 \\
$r_{k}^{\text {Lin }}$ & 0.0700 & 0.8400 & 0.9100 & 0.9800 & 1.0500 & 1.1200 \\
$r_{k}^{\text {Opt }}$ & 0.6851 & 0.7786 & 0.8721 & 0.9656 & 1.0591 & 1.1526 \\
\hline
\end{tabular}

\section{Acknowledgments}

The authors are grateful to the editor and the anonymous referees for their helpful comments and suggestions, which have helped them produce a substantially improved version. Their work is supported by National Science Foundation of China (Grant no. 11171321).

\section{References}

[1] R. Norberg, "A credibility theory for automobile bonus systems," Scandinavian Actuarial Journal, no. 2, pp. 92-107, 1976.

[2] J. Lemaire and H. Zi, "A comparative analysis of 30 bonus-malus systems," ASTIN Bulletin, vol. 24, no. 2, pp. 287-309, 1994.

[3] J. Lemaire, Bonus-malus Systems in Automobile Insurance, Kluwer Academic, Boston, Mass, USA, 1995.

[4] J. F. Walhin and J. Paris, "Using mixed Poisson distribution in connection with bonus-malus system," ASTIN Bulletin, vol. 29, pp. 81-99, 1999.

[5] N. Frangos and S. Vrontos, "Design of optimal bonus-malus system with a frequency and a severity component on an individual basis in automobile insurance," ASTIN Bulletin, vol. 31, pp. 1-23, 2001.

[6] R. Mahmoudvand and H. Hassani, "Generalized bonus-malus systems with a frequency and a severity component on an individual basis in automobile insurance," Astin Bulletin, vol. 39, no. 1, pp. 307-315, 2009.

[7] V. Gilde and B. Sundt, "On bomus systems with credibility scales," Scandinavian Actuarial Journal, pp. 13-22, 1999.

[8] K. Loimaranta, "Some asymptotic properties of bonus systems," ASTIN Bulletin, vol. 6, pp. 223-245, 1972.

[9] M. Denuit, X. Marechal, S. Pitrebois, and J. F. Walhin, Acturial Modilling of Claim Counts: Risk Classification, Credibility and Bomus-Malus Systems, Wiley, 2007. 


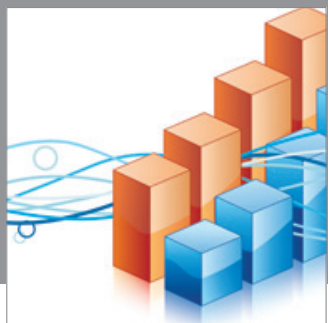

Advances in

Operations Research

mansans

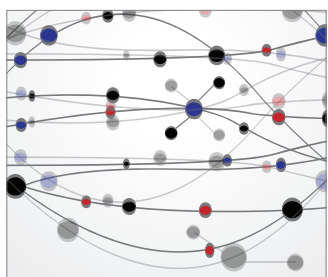

The Scientific World Journal
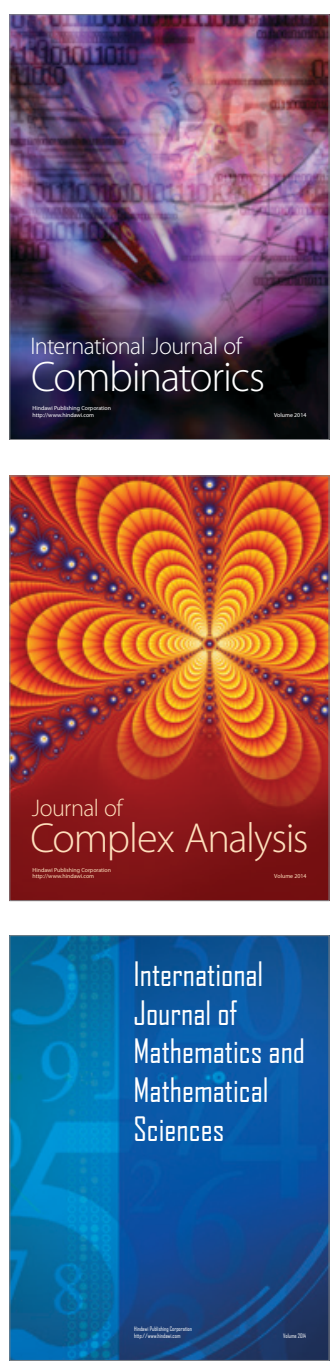
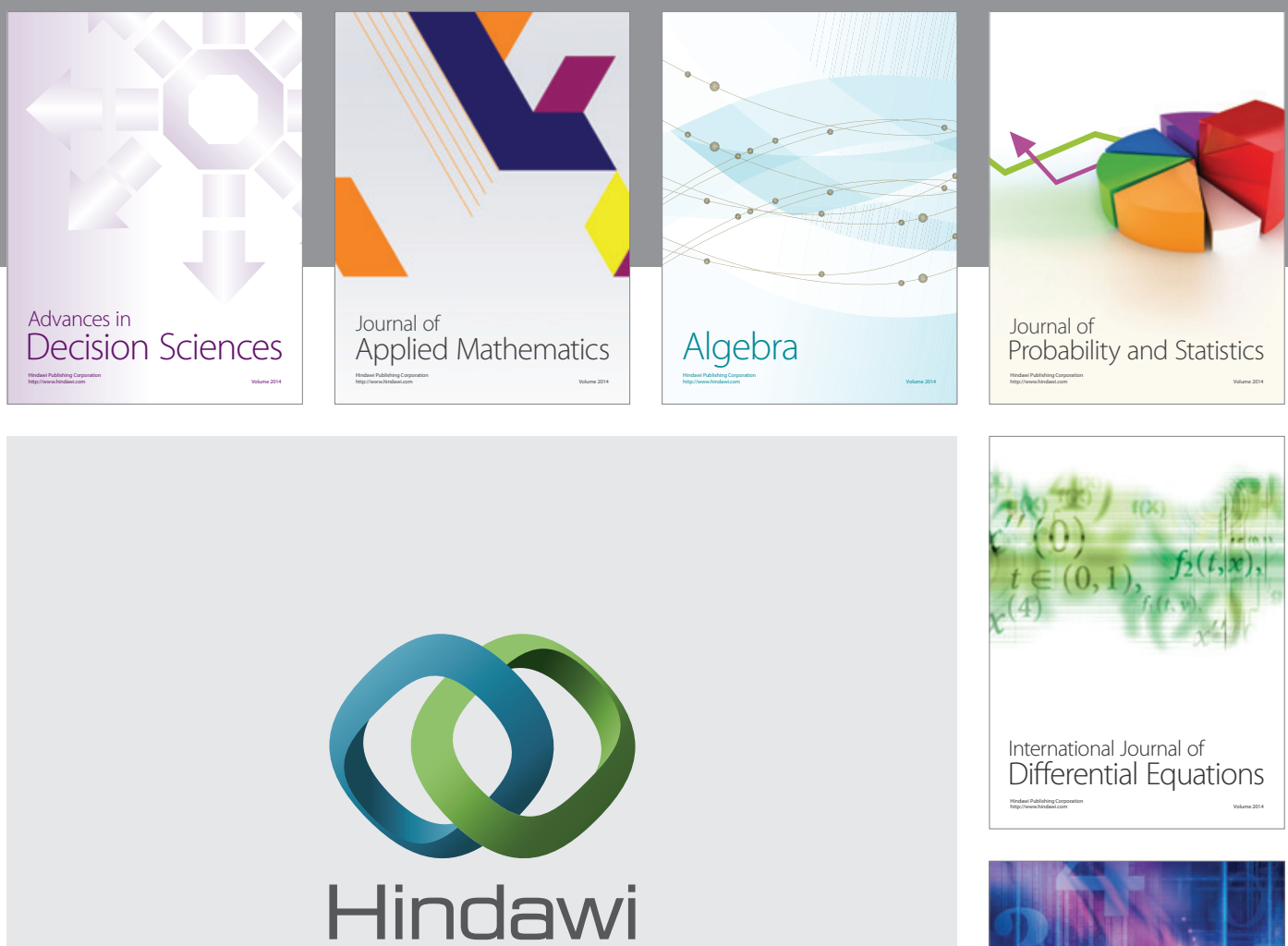

Submit your manuscripts at http://www.hindawi.com
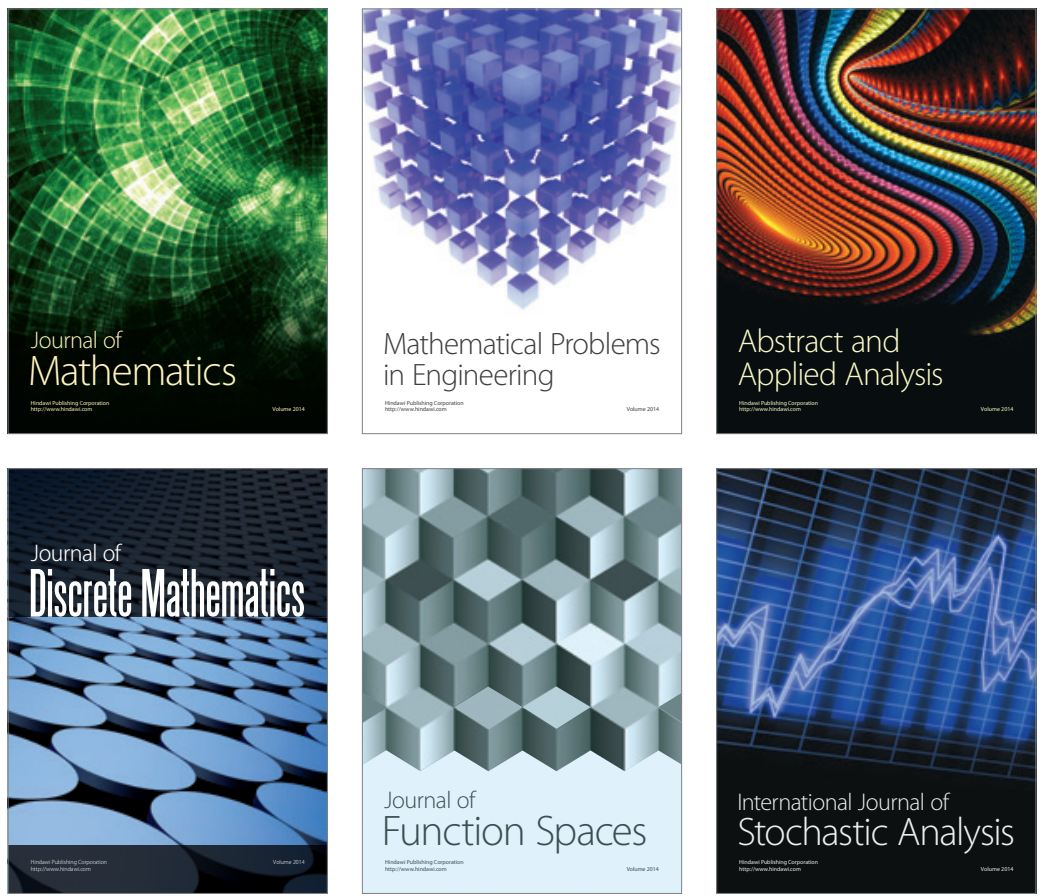

Journal of

Function Spaces

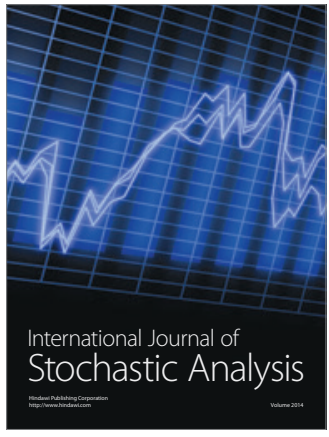

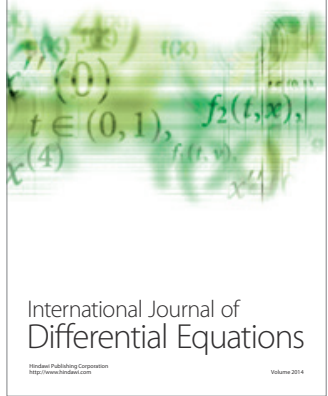
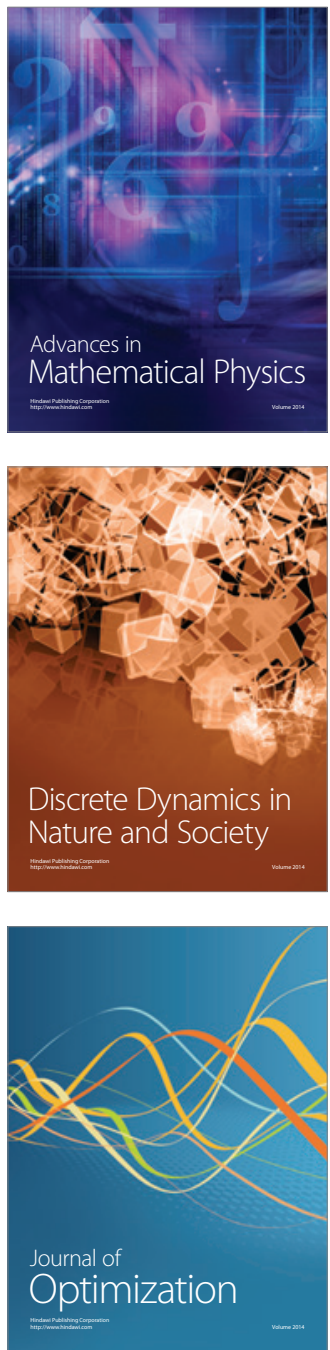